\title{
SELETIVIDADE DE ACARICIDAS AOS PREDADORES Scymnus sp. (Coleoptera: Coccinelidae) E ARANEAE (Arachnida) EM ALGODOEIRO (Gossypium hirsutum L.) SOB CONDIÇÕES DE CAMPO
}

\author{
RENATO SUEKANE* \\ PAULO EDUARDO DEGRANDE** \\ IZIDRO DOS SANTOS DE LIMA JUNIOR* \\ CÁSSIO KODAMA*** \\ EVERTON KODAMA****
}

\begin{abstract}
Neste trabalho avaliou-se a seletividade dos acaricidas utilizados na cultura do algodão sobre os predadores Scymnus sp. e Araneae, em condições de campo, e de acordo com a classificação da IOBCwprs. Também foram estudadas as porcentagens de mortalidade e a duração da atividade tóxica dos tratamentos, mediante delineamento experimental em blocos ao acaso, com onze tratamentos e quatro repetições. A análise da composição da artropodofauna total indicou que os "inimigos naturais" mais abundantes ocorrentes no estudo foram o coccinelídeo Scymnus sp. (58\% do total) e a ordem Araneae (24\%). Talstar ${ }^{\circledR} 100$ EC e Danimen ${ }^{\circledR} 300$ EC foram classificados como prejudiciais à Scymnus sp. e Araneae, com duração da atividade tóxica superior a 12 dias. Kumulus ${ }^{\circledR} 800$ WG, Oberon $^{\circledR} 240$ SC, Borneo ${ }^{\circledR} 110$ SC e MilbeKnock ${ }^{\circledR} 50$ EC mostraramse inócuos à joaninha Scymnus sp., e sem atividade tóxica já no primeiro dia após a aplicação. Para Araneae, os produtos Kumulus ${ }^{\circledR}$ 800 WG, Oberon ${ }^{\circledR} 240$ SC, Borneo ${ }^{\circledR} 110$ SC e MilbeKnock ${ }^{\circledR} 50$ EC mostraram-se moderadamente prejudiciais. Pirate ${ }^{\circledR} 240$ SC, Polo ${ }^{\circledR}$ 500 SC, Acarit $^{\circledR} 720$ EC e Vertimec ${ }^{\circledR} 18$ EC foram moderadamente prejudiciais para ambos os táxons.
\end{abstract} $N \tilde{O} O$ - ALVO.

\footnotetext{
* Engenheiros Agrônomos, Doutorandos, Programa de Pós-Graduação em Agronomia, Universidade Federal da Grande Dourados (UFGD), Faculdade de Ciências Agrárias (FCA), Dourados, MS (e-mail: rsuekane@hotmail.com, izidro. lima@ifms.edu.br).

** Engenheiro Agrônomo, Doutor em Ciências Biológicas (Zoologia), Professor Associado, Entomologia Agrícola, UFGD/ FCA, Dourados, MS (e-mail: paulodegrande@ufgd.edu.br).

*** Engenheiro Agrônomo, Mestrando, Programa de Pós-Graduação em Agronomia, UFGD/FCA, Dourados, MS (e-mail: cassio_kodam@hotmail.com).

**** Graduando em Agronomia, UFGD/FCA, Dourados, MS (e-mail: evertonkodama@hotmail.com).
} 


\section{INTRODUÇÃo}

Atitudes que mantêm os predadores de artrópodes pragas nos agroecossistemas são fundamentais para o equilíbrio dinâmico de suas populações e para o equilíbrio biológico na cultura do algodão. $O$ uso de produtos seletivos é de grande importância para minimizar os problemas de ressurgência e surtos de pragas secundárias (CROCOMO, 1984). Assim, há a necessidade de serem identificados e utilizados os pesticidas comprovadamente seletivos para a atuação dos agentes de controle biológico no Manejo Integrado de Pragas (MIP). As pesquisas sobre seletividade a campo com artrópodes não alvo costumam ser caras, complexas e os resultados surpreendentemente simplistas, cuja experiência prática indica que os dados devem ser interpretados ao nível taxonômico (BROWN, 2004).

A joaninha Scymnus sp. (SILVIE et al., 2001) e as aranhas (RAMALHO et al., 1990) atuam como importantes predadores de lepidópteros e pulgões. Gravena (1990) citou os predadores mais importantes e abundantes na cultura do algodão, incluindo percevejos (Orius sp. e Nabis sp.), coccinelídeos (Coleomegilla maculata, Scymnus, Cycloneda sanguinea e Eriopsis conexa), carabídeos (Calosoma granulatum, Lebia concinna e Callida spp.), crisopídeos (Chrysoperla carnea, C. externa, Chrysopa bicarnea e Ceraeochrysa cubana), formigas (Solenopsis invicta), tesourinhas (Doru lineare), aranhas (Araneae) Oxyopes salticus, Misumenops sp., Chicaranthium sp., Ancanthepeira stellata, Tetragnatha laboriosa, Aysha gracilis, Phidippus audax, Pardosa sp., Theridula gonygaster e Chrysso clementinae. Por outro lado, o ácaro-rajado Tetranychus urticae (Acari: Tetranychidae) e o ácaro-branco Polyphagotarsonemus latus (Acari: Tarsonemidae) estão listados como importantes fitófagos do algodoeiro e para seu controle normalmente são usados acaricidas. Os principais ingredientes ativos utilizados para o controle dos ácaros na cultura do algodão são abamectina, bifentrina, clorfenapir, diafentiuron, enxofre, espiromesifeno, etoxazol, fenpropatrina, milbemectina e propargito (ANDREI, 2005).

Estudos de seletividade de pesticidas aos principais inimigos naturais de pragas geram informações práticas que são utilizadas na tomada de decisão com relação ao produto a ser escolhido para controlar determinada praga. Segundo Brown (2004), os estudos de campo tipicamente geram informações da abrangência dos táxons afetados como proporção da fauna amostrada que foi reduzida pela aplicação do pesticida, a magnitude dos efeitos (muitas vezes expressa pela redução percentual da quantidade amostrada), e a duração de tais efeitos (tempo necessário para a recuperação ocorrer). A classificação da seletividade deve ser feita pelo nome do produto comercial, uma vez que o mesmo ingrediente ativo pode estar em distintas formulações comerciais e ocasionar impacto diferenciado sobre o inseto-teste (HASSAN et al., 2000). Barros et al. (2003) identificaram que o melhor método para a amostragem de predadores para estudos de seletividade é o de batida de pano, que apresenta vantagens como: ser menos monótono, simples, prático e rápido. Esse método possibilita a coleta de maior diversidade de inimigos naturais, mas com o inconveniente de não amostrar ovos e pupas.

O presente estudo teve por objetivo avaliar o impacto dos acaricidas químicos utilizados no controle dos ácaros $T$. urticae e $P$. latus sobre o complexo de inimigos naturais ocorrentes na parte aérea do algodoeiro, com ênfase nos táxons abundantes.

\section{MATERIAL E MÉTODOS}

O trabalho foi realizado na Faculdade de Ciências Agrárias (FCA) da Universidade Federal da Grande Dourados (UFGD), localizada no município de Dourados, Mato Grosso do Sul (22 $13^{\prime} 16^{\prime \prime}$ S e 54 $4^{\circ} 7^{\prime} 01^{\prime \prime}$ W) a $430 \mathrm{~m}$ de altitude. O solo dessa área é classificado como Latossolo Vermelho distroférrico, de textura argilosa. Área com 0,9 ha de algodão foi cultivada para o estudo, mediante 
preparo convencional do solo, sendo semeado o cultivar NuOpal Bollgard ${ }^{\circledR}$, em 10/1/2008, com estande de dez plantas por metro linear e espaçamento de $0,90 \mathrm{~m}$ entre linhas. Realizou-se o controle de plantas daninhas mediante aplicação de herbicidas pré e pós-emergente. Não houve controle químico das pragas e doenças foliares em nenhuma etapa do desenvolvimento da cultura, pois visou-se estabelecer a população de inimigos naturais das pragas do algodoeiro. Segundo Sujii et al. (2007), a abundância de aranhas e de insetos e ácaros predadores seria menor caso a população de presas herbívoras fosse reduzida, o que poderia potencialmente interferir na diversidade da cadeia alimentar.

Como alguns estudos mostraram que no Mato Grosso do Sul (BARROS et al., 2006) e em São Paulo (CAMPOS et al., 1986; RAMIRO e FARIA, 2006) foram observadas maiores abundâncias de predadores e suas associações com as populações do pulgão Aphis gossypii Glover, após o aparecimento dos primeiros botões florais, semanalmente foram realizadas dez batidas de pano (BARROS et al., 2003) para o acompanhamento da ocorrência de inimigos naturais. Após a colonização do ambiente pelos organismos benéficos, realizou-se a aplicação dos acaricidas por meio de pulverizador de barra de pressão constante, com propulsão à base de gás carbônico, dotado de bicos do tipo cone vazio JA preto. Obedeceu-se a diluição de 110 litros de calda por hectare, sendo rigorosamente respeitadas as condições ideais de aplicação dos produtos no que tange à umidade relativa do $\operatorname{ar}(>60 \%)$ e velocidade do vento $(<4 \mathrm{~km} / \mathrm{h})$.

Adotou-se o delineamento experimental de blocos ao acaso, com 11 tratamentos e quatro repetições. Cada parcela foi constituída por quinze linhas da cultura com quinze metros de comprimento. Os acaricidas estudados nas suas dosagens agronômicas registradas para a cultura encontram-se descritos na Tabela 1.

Para quantificar os predadores, discriminando-os por táxon, foram realizadas seis avaliações: uma prévia à aplicação dos acaricidas e em 1, 3, 6, 9 e 12 dias após o tratamento (dat), usando-se o método de batida de pano, com dimensões de um metro de comprimento por 0,90 m de largura e amostrando-se a quantidade de inimigos naturais em duas linhas da cultura e em cinco pontos aleatórios dentro da parcela. Essas avaliações sempre foram realizadas nas onze linhas centrais da unidade experimental, desconsiderando-se dois metros em cada extremidade como bordadura, visando evitar possíveis interferências de parcelas vizinhas. Quando não foi possível identificar os insetos a campo, esses eram levados para o laboratório para se proceder a identificação amparada em literatura específica.

Os dados foram submetidos à análise de variância (Anova) e ao teste $\mathrm{F}$ de significância $(\alpha=0,01$ e 0,05$)$. Como proposto por Gomes (1982) quando $F$ calculado foi maior que o $F$ tabelado aplicou-se o teste de Tukey aos níveis de 1 a $5 \%$ de probabilidade, obtendo-se as diferenças mínimas significativas entre os tratamentos. As análises estatísticas e os cálculos de seletividade foram efetuados com os inimigos naturais mais abundantes e o complexo total, dado pelo somatório dos predadores nas parcelas. Para o cálculo da porcentagem de mortalidade dos acaricidas testados utilizou-se a fórmula de Henderson e Tilton (1955). Posteriormente, os acaricidas foram classificados quanto a magnitude dos efeitos, expressa pela redução percentual da quantidade amostrada (mortalidade) devido à aplicação do pesticida, usando-se a escala do IOBCwprs (International Organization for Biological and Integrated Control, West Palaearctic Regional Section), citada por Boller et al. (2005). Nessa escala, o produto inócuo, inofensivo, que não causa danos consideráveis à população, ou é levemente prejudicial (classe $\mathrm{N}$ ) reduz de $0-50 \%$ os inimigos naturais; o produto moderadamente prejudicial (classe $\mathrm{M}$ ) reduz de $50-75 \%$ os inimigos naturais; e o produto prejudicial, nocivo ou que causa danos consideráveis (classe T) alcança $>75 \%$ de redução da população de inimigos naturais. Também foi estabelecida a duração da atividade tóxica, chamada de período de restabelecimento ou recuperação por Brown (2004), mediante análise do tempo necessário para a população atingir a classe $\mathrm{N}$ após a aplicação. 
TABELA 1 - NOME DO PRODUTO COMERCIAL (pc), INGREDIENTE ATIVO (ia), GRUPO PRINCIPAL E SÍTIO DE AÇÃO PRIMÁRIO BASEADO NO MODO DE AÇÃO, SUBGRUPO QUÍMICO (IRAC, 2011) E DOSAGEM DO PRODUTO COMERCIAL UTILIZADA POR HECTARE (mL ou g. ha')

\begin{tabular}{|c|c|c|c|c|}
\hline Tratamento (pc) & la & $\begin{array}{l}\text { Grupo principal } \\
\text { I Sítio de ação } \\
\text { primário }\end{array}$ & $\begin{array}{l}\text { Subgrupo químico ou ia } \\
\text { exemplificador }\end{array}$ & $\begin{array}{c}\text { mL ou } \\
\text { g do pc } \\
\text { ha-1 }\end{array}$ \\
\hline 1 Talstar $^{\circledR} 100$ EC & Bifentrina & $\begin{array}{l}\text { Modulador de canal } \\
\text { de sódio }\end{array}$ & Piretroide $(3 A)$ & 600 \\
\hline 2 Pirate ${ }^{\circledR} 240$ SC & Clorfenapir & $\begin{array}{l}\text { Desacoplador } \\
\text { da fosforilação } \\
\text { oxidativa via ruptura } \\
\text { do gradiente } \\
\text { de prótons - } \\
\text { metabolismo } \\
\text { energético }\end{array}$ & $\begin{array}{l}\text { Clorfenapir, DNOC, } \\
\text { Sulfluramido (13) }\end{array}$ & 1000 \\
\hline $3 \mathrm{Polo}^{\circledR} 500 \mathrm{SC}$ & Diafentiurom & $\begin{array}{l}\text { Inibidor de ATP } \\
\text { sintase mitocondrial } \\
\text { - metabolismo de } \\
\text { energia }\end{array}$ & Feniltioureia $\left(12^{a}\right)$ & 800 \\
\hline 4 Kumulus $^{\circledR} 800$ WG & Enxofre & $\begin{array}{l}\text { Oxida a ácido } \\
\text { sulfuroso - redução } \\
\text { do } \mathrm{S} \text { para } \mathrm{H}_{2} \mathrm{~S}\end{array}$ & Miscelânea, inorgânico (8) & 600 \\
\hline 5 Oberon $^{\circledR} 240$ SC & Espiromesifeno & $\begin{array}{l}\text { Inibidor da acetil- } \\
\text { CoA carboxilase - } \\
\text { síntese de lipídios, } \\
\text { regulação do } \\
\text { crescimento }\end{array}$ & $\begin{array}{l}\text { Derivados dos ácidos } \\
\text { tetrônico e tetrâmico (23) }\end{array}$ & 600 \\
\hline 6 Borneo $^{\circledR} 110$ SC & Etoxazol & $\begin{array}{l}\text { Inibidor de } \\
\text { crescimento de } \\
\text { ácaros }\end{array}$ & Etoxazol (10B) & 230 \\
\hline 7 Danimen $^{\circledR} 300$ EC & Fenpropatrina & $\begin{array}{l}\text { Modulador de canal } \\
\text { de sódio }\end{array}$ & Piretroide $(3 A)$ & 400 \\
\hline $\begin{array}{l}8 \text { MilbeKnock }^{\circledR} 50 \\
\text { EC }\end{array}$ & Milbemectina & $\begin{array}{l}\text { Ativador do canal } \\
\text { de cloro - ação no } \\
\text { nervo e músculo }\end{array}$ & Milbemicinas (6) & 240 \\
\hline 9 Acarit $^{\circledR} 720$ EC & Propargito & $\begin{array}{l}\text { Inibidor de ATP } \\
\text { sintase mitocondrial } \\
\text { - metabolismo de } \\
\text { energia }\end{array}$ & Propargito (12C) & 1500 \\
\hline 10 Vertimec $^{\circledR} 18$ EC & Abamectina & $\begin{array}{l}\text { Ativador do canal } \\
\text { de cloro - ação no } \\
\text { nervo e músculo }\end{array}$ & Avermectina (6) & 600 \\
\hline $\begin{array}{l}11 \text { Testemunha } \\
\text { (água) }\end{array}$ & - & - & - & - \\
\hline
\end{tabular}

\section{RESULTADOS E DISCUSSÃO}

A análise da composição da artropodofauna (Figura 1) indicou que os mais abundantes inimigos naturais ocorrentes no estudo, expressos em porcentagem de indivíduos por táxon em 
relação ao total de indivíduos amostrados, foram: o coccinelídeo Scymnus sp. (58\%), a ordem Araneae (24\%), os demais Coccinellidae (10\%), o Geocoris sp. (4\%) e a família Syrphidae (3\%). Foram denominados como "outros" (1\%) aqueles inimigos naturais encontrados em menor quantidade, decorrentes do somatório de Podisus sp., Forficulidae, Chrysopidae, Braconidae, Reduviidae e Mantispidae.

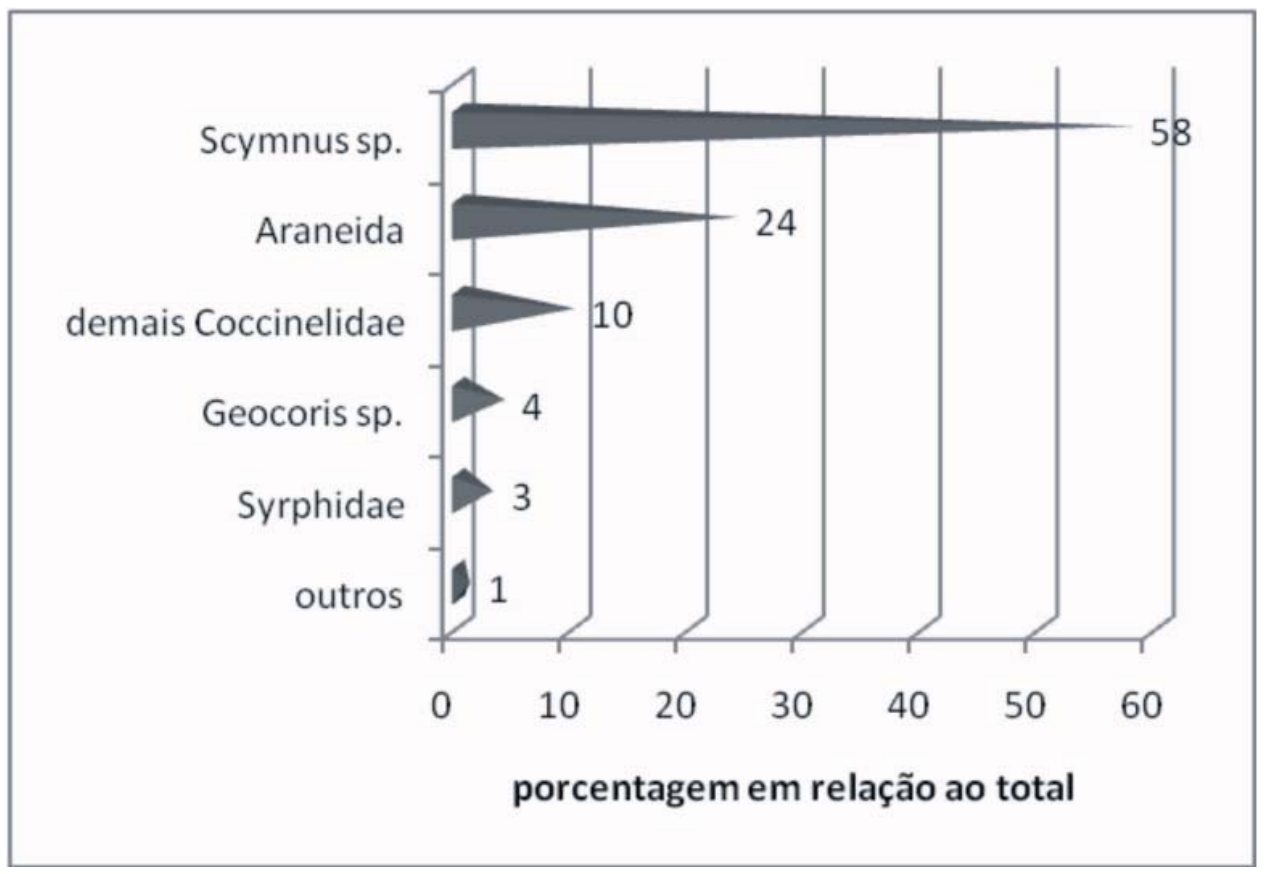

\section{FIGURA 1 - COMPOSIÇÃO DA ARTROPODOFAUNA-TESTE. GRUPOS TAXONÔMICOS DE INIMIGOS NATURAIS MAIS ABUNDANTES EM PORCENTAGEM DE INDIVÍDUOS EM RELAÇÃO AO TOTAL AMOSTRADO - DOURADOS/2008}

Dentre os insetos da família Coccinelidae, o gênero Scymnus sp. foi o mais abundante, corroborando os resultados de Barros et al. (2006), que estudaram a flutuação populacional de insetos predadores associados às pragas do algodoeiro. Verificam que o gênero Scymnus sp. representou $36,5 \%$ e Araneae $8 \%$ do total amostrado. Campos et al. (1986) constataram que Scymnus sp. foi o predador mais abundante nos algodoeiros estudados, representado $48,2 \%$ da população em relação aos demais artrópodes e 74,7\% em relação aos demais coccinelídeos. Ribeiro (2007), em Mato Grosso do Sul, e Nunes (1999) em Goiás, realizaram levantamento de inimigos naturais na cultura do algodão e identificaram indivíduos dos mesmos grupos taxonômicos encontrados no presente trabalho, porém notaram outra proporção relativa entre os artrópodes da composição faunística. Scymnus sp., Araneae e o somatório dos indivíduos dos diferentes táxons (expresso na terminologia "complexo") foram considerados na análise dos resultados.

\subsection{SELETIVIDADE À Scyomnus sp.}

Na Tabela 2 encontram-se todos os resultados dos tratamentos com acaricidas sobre o predador Scymnus sp. A avaliação prévia à aplicação dos acaricidas evidenciou infestação de Scmynus sp., variando de 2,5 a 8,25 indivíduos em média por parcela, e a análise estatística (estudo da variância e teste $F$ de significância com $\alpha=0,05$ ) indicou população uniforme na área por ocasião da aplicação dos tratamentos. Apesar dessa uniformidade, a mortalidade foi calculada pela fórmula de Henderson \& Tilton (1955), que pondera a população existente nas parcelas dos tratamentos químicos sob análise e na testemunha, antes e depois da aplicação. 
A avaliação realizada 1 dia após o tratamento (dat) permitiu classificar Talstar ${ }^{\circledR} 100 \mathrm{EC}$ e Danimen ${ }^{\circledast} 300$ EC como prejudiciais à população de Scymnus sp. (classe T, provoca mortalidade superior a 75\%), segundo a IOBCwprs (BOLLER et al. 2005), capazes de causar danos na população desse predador em função do efeito de choque. Polo ${ }^{\circledast} 00$ SC, Acarit ${ }^{\circledR} 720$ EC e Vertimec $^{\circledR} 18$ EC enquadraram-se na classe $\mathrm{M}$, moderadamente prejudicial, a qual contempla os pesticidas que provocam mortalidade entre $50-75 \%$. Os demais acaricidas foram considerados inofensivos ou levemente prejudiciais à Scymnus sp. (classe $\mathrm{N}$ ).

Aos 3 dat, os piretróides Talstar ${ }^{\circledR} 100$ EC e Danimen ${ }^{\circledR} 300$ EC permaneceram na classe T e Vertimec ${ }^{\circledR} 18$ EC se manteve na classe M. Os acaricidas Polo ${ }^{\circledR} 500$ SC e Acarit ${ }^{\circledR} 720$ EC tiveram seus efeitos nocivos reduzidos, mudando para as classes $\mathrm{M}$ e N , respectivamente. Os demais acaricidas testados continuaram categorizados como inofensivos ou pouco prejudiciais (classe $\mathrm{N}$ ) à Scymnus sp.

Aos 6 dat, Talstar ${ }^{\circledR} 100$ CE manteve-se na classe T. Vertimec ${ }^{\circledR} 18$ EC permaneceu na classe M, junto do Pirate ${ }^{\circledR} 240$ SC, que manifestou sua maior toxicidade à Scymnus sp. nessa avaliação. Os demais tratamentos foram considerados como inócuos ou levemente prejudiciais (classe $\mathrm{N}$ ), inclusive o Danimen ${ }^{\circledR} 300 \mathrm{EC}$ que mostrou a menor duração da atividade tóxica em relação ao também piretróide Talstar ${ }^{\circledR} 100 \mathrm{CE}$.

$\mathrm{Na}$ avaliação realizada aos 9 dat, Talstar ${ }^{\circledR} 100 \mathrm{EC}$ manteve-se na classe T, sendo que os demais acaricidas mostraram-se inócuos (classe N). Na última avaliação, aos 12 dat, Talstar ${ }^{\circledR} 100$ EC migrou da classe T para M, com 69,64\% de mortalidade de Scymnus sp. Os demais produtos foram inofensivos (classe $\mathrm{N}$ ) para esse coccinelídeo.

Kumulus $^{\circledR} 800$ WG, Oberon ${ }^{\circledR} 240$ SC, Borneo ${ }^{\circledR} 110$ SC e MilbeKnock ${ }^{\circledR} 50$ EC foram altamente seletivos ao Scymnus sp., sendo categorizados como inócuos ou levemente nocivos (classe $\mathrm{N}$ ) ao longo de todo o estudo.

Os resultados da duração da atividade tóxica são importantes para se entender a recolonização de área tratada por determinada espécie em razão de fornecer informações para programas de MIP, especialmente no que se refere à compatibilização de tratamentos químicos com liberações programadas de inimigos naturais. Neste experimento, Talstar ${ }^{\circledR} 100 \mathrm{EC}$ foi o inseticida que causou maiores danos à população de Scymnus sp., com duração da atividade tóxica entre 9 e 12 dat, a mais longa entre os acaricidas testados. Danimen ${ }^{\circledR} 300$ EC também foi prejudicial ao predador, porém a duração da sua atividade tóxica (classe T) foi menor que a do $\operatorname{Talstar}^{\circledR} 100$ EC, ficando entre 3 e 6 dat. Apesar da IOBCwprs (BOLLER et al., 2005) não recomendar estudos estatísticos dos resultados para a categorização, levando em conta apenas a mortalidade, os dados de Scymnus sp. foram submetidos á análise de variância e ao teste $\mathrm{F}$ de significância (com $\alpha=$ $99 \%$ ). A diferença estatisticamente significativa entre as médias dos tratamentos acaricidas e a testemunha ocorreu para o efeito de choque do Talstar ${ }^{\circledR} 100 \mathrm{EC}$, evidenciando ação inicial e rápida do produto na população dessa joaninha.

\subsection{SELETIVIDADE À ARANEAE}

A avaliação prévia à aplicação dos acaricidas (Tabela 3 ) indicou infestação média de Araneae, variando de 3,5 a 7 indivíduos por parcela, cujos dados analisados estatisticamente (pelo estudo da variância e teste $F$ de significância com $\alpha=0,05$ ) indicaram população uniforme na área por ocasião do tratamento. A mortalidade desses indivíduos também foi calculada pela fórmula de Henderson \& Tilton (1955).

A análise da seletividade dos acaricidas sobre as aranhas, Tabela 3 , indicou que na avaliação realizada a 1 dat o piretroide Talstar ${ }^{\circledR} 100$ EC promoveu a maior mortalidade, com $84,92 \%$, sendo classificado como prejudicial $(\mathrm{T})$. Seu efeito de choque foi caracterizado pela análise estatística (teste Tukey $1 \%$ ), mediante diferença significativa em relação à testemunha. Os produtos Pirate ${ }^{\circledR} 240$ SC, Polo $^{\circledast} 500$ SC, Oberon ${ }^{\circledR} 240$ SC, Borneo ${ }^{\circledast} 110$ SC, Danimen ${ }^{\circledast} 300$ EC, MilbeKnock ${ }^{\circledast} 50$ EC, Acarit ${ }^{\circledR} 720$ EC e Vertimec ${ }^{\circledR} 18 \mathrm{EC}$ foram enquadrados na classe $\mathrm{M}$ (moderadamente prejudicial), enquanto 0 Kumulus $^{\circledR} 800$ WG foi classificado como inofensivo a levemente prejudicial à Araneae. Kumulus ${ }^{\circledR} 800$ WG, Vertimec ${ }^{\circledast} 18 \mathrm{EC}$ e Acarit $720 \mathrm{EC}$ mostraram-se estatisticamente semelhantes à testemunha, ao nível de $1 \%$ de probabilidade, na primeira avaliação da atividade tóxica dos acaricidas. 


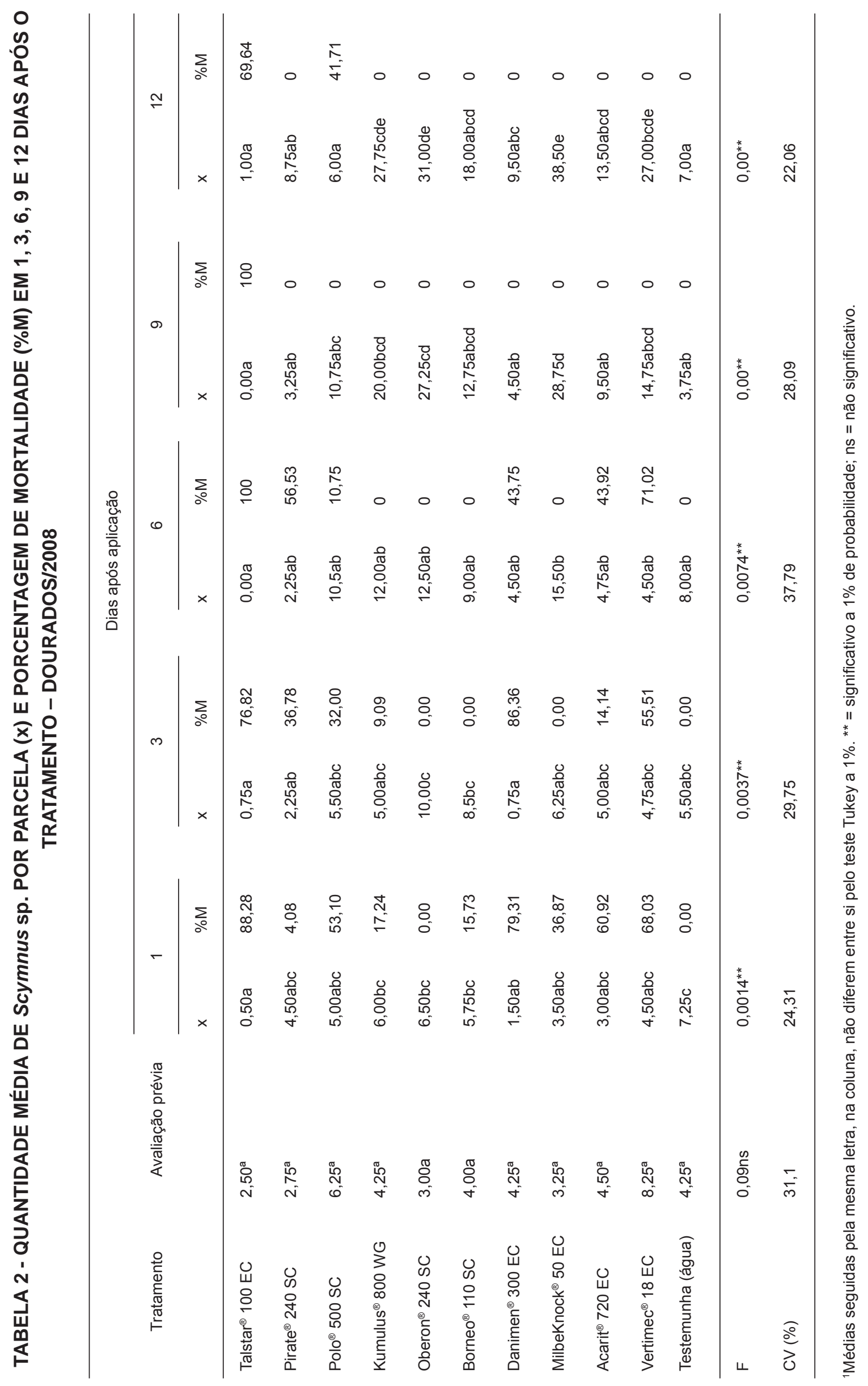


Na avaliação realizada aos 3 dias após o tratamento Danimen ${ }^{\circledR} 300$ EC passou da classe $\mathrm{M}$ para a $\mathrm{T}\left(86,26 \%\right.$ de mortalidade). Talstar ${ }^{\circledR} 100 \mathrm{EC}$ permaneceu na mesma classe $(\mathrm{T})$ de seletividade às aranhas. Pirate ${ }^{\circledR} 240$ SC, Polo 500 SC, Borneo ${ }^{\circledR} 110$ SC e Acarit ${ }^{\circledR} 720$ EC permaneceram na classe M, mas Oberon ${ }^{\circledR} 240$ SC, MilbeKnock ${ }^{\circledR} 50$ EC e Vertimec ${ }^{\circledR} 18$ EC migraram para o menor nível de toxicidade da classe N. Kumulus ${ }^{\circledR} 800$ WG continuou inofensivo de acordo com essa classificação.

Aos 6 dias após o tratamento, os piretroides Talstar $^{\circledR} 100$ EC e Danimen ${ }^{\circledR} 300$ EC permaneceram na classe T, sendo prejudiciais para Araneae. Polo ${ }^{\circledR} 500$ SC, Acarit ${ }^{\circledR} 720$ EC e Vertimec $^{\circledR} 18 \mathrm{EC}$ foram categorizados na classe M, enquanto o Pirate ${ }^{\circledR} 240$ SC migrou da classe M para a classe $\mathrm{N}$ em relação à avaliação anterior. Os demais acaricidas continuaram na classe $\mathrm{N}$ de seletividade.

Na avaliação aos 9 dat, Talstar ${ }^{\circledR} 100$ EC e Danimen ${ }^{\circledR} 300$ EC (que nas avaliações anteriores estavam na classe $\mathrm{T}$ ) foram classificados como $\mathrm{M}$, indicando o início da perda de suas atividades tóxicas. Kumulus ${ }^{\circledR} 800$ WG e Borneo ${ }^{\circledR} 110$ SC mostraram aumento na mortalidade sem explicação plausível em relação aos resultados desse experimento e se posicionaram na classe M. Ficaram na classe N os seguintes produtos: Pirate ${ }^{\circledast} 240$ SC, Polo ${ }^{\circledast} 500$ SC, Oberon ${ }^{\circledast} 240$ SC, MilbeKnock ${ }^{\circledast} 50$ $\mathrm{EC}$, Acarit $^{\circledR} 720 \mathrm{EC}$ e Vertimec $^{\circledR} 18 \mathrm{EC}$.

$\mathrm{Na}$ avaliação aos 12 dias após o tratamento, Talstar ${ }^{\circledR} 100 \mathrm{EC}$ voltou a ser classificado como $\mathrm{T}$, indicando sua duradoura atividade tóxica. Danimen ${ }^{\circledR} 300 \mathrm{EC}$ e Acarit ${ }^{\circledR} 720 \mathrm{EC}$ foram classificados como M, com aumento na toxicidade desse último, também circunstancial. Kumulus ${ }^{\circledR} 800$ WG voltou à condição de classe $\mathrm{N}$, assim como os demais tratamentos. Estatisticamente, as parcelas de todos os tratamentos se igualaram à testemunha na última avaliação. Tal fato sugere a recolonização das parcelas pelos inimigos naturais (Tabela 3), exceção ao tratamento Talstar ${ }^{\circledR} 100 \mathrm{EC}$ que continuou afetando as aranhas em suas parcelas.

\subsection{SELETIVIDADE AO COMPLEXO}

Os resultados relativos à ação dos acaricidas no complexo de artrópodes ocorrentes (Tabela 4) foram influenciados pelos efeitos sobre Scymnus sp. e Araneae, os táxons mais abundantes no estudo em função das suas contribuições relativas nas médias totalizadas. Avaliando a mortalidade para o complexo de inimigos naturais ocorrentes na área experimental, em 1 dat, Talstar ${ }^{\circledR} 100$ EC e Danimen ${ }^{\circledR} 300$ EC foram enquadrados na classe T. Pirate ${ }^{\circledR} 240$ SC, Polo $^{\circledR} 500$ SC, Borneo ${ }^{\circledR} 110$ SC, MilbeKnock ${ }^{\circledR} 50$ EC, Acarit ${ }^{\circledR} 720$ EC e Vertimec ${ }^{\circledR} 18$ EC foram classificados como M. Kumulus ${ }^{\circledR} 800$ WG e Oberon $^{\circledR} 240$ SC mostraram-se pouco tóxicos ou inofensivos nessa data.

Aos 3 dias após o tratamento, Talstar $^{\circledR} 100$ EC e Danimen ${ }^{\circledR} 300$ EC permaneceram na mesma classe $(T)$, sendo nocivos aos predadores ocorrentes. Pirate ${ }^{\circledR} 240$ SC e Vertimec $^{\circledR} 18$

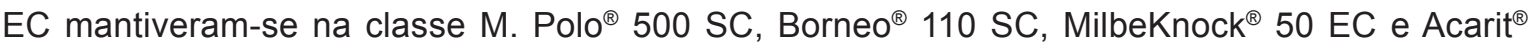
720 EC foram classificados como N, ficando na mesma classe de Kumulus ${ }^{\circledR} 800$ WG e Oberon ${ }^{\circledR}$ 240 SC.

$\mathrm{Na}$ avaliação aos 6 dat, Talstar $^{\circledR} 100 \mathrm{EC}$ permaneceu na classe T. Os acaricidas enquadrados na classe M foram Pirate ${ }^{\circledR} 240$ SC, Danimen ${ }^{\circledR} 300$ EC e Vertimec ${ }^{\circledR} 18$ EC, sendo os demais classificados como N. Aos 9 dat, Talstar ${ }^{\circledast} 100 \mathrm{EC}$ seguiu na mesma classificação anterior (classe $\mathrm{T}$ ), enquanto que o Danimen ${ }^{\circledR} 300 \mathrm{EC}$, com atividade tóxica reduzida na amostragem anterior, continuou na classe M. Os demais pesticidas mostraram-se pouco tóxicos (classe $\mathrm{N}$ ) ao complexo de inimigos naturais. Aos 12 dias após o tratamento, apenas Talstar ${ }^{\circledR} 100$ EC foi classificado como $\mathrm{T}$, sendo os demais produtos inofensivos ou levemente prejudiciais ao complexo de antrópodes benéficos estudados (Tabela 4). 


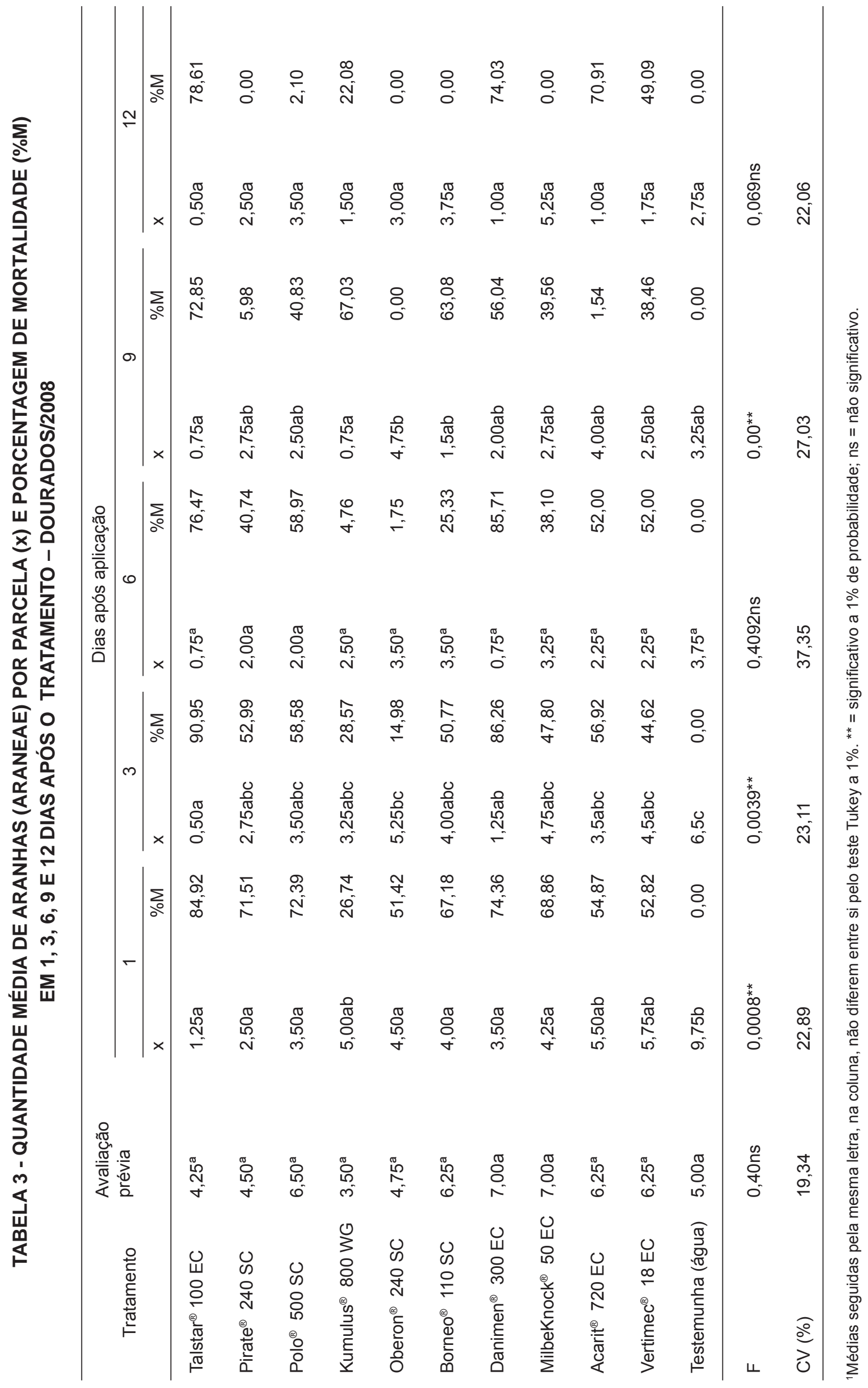




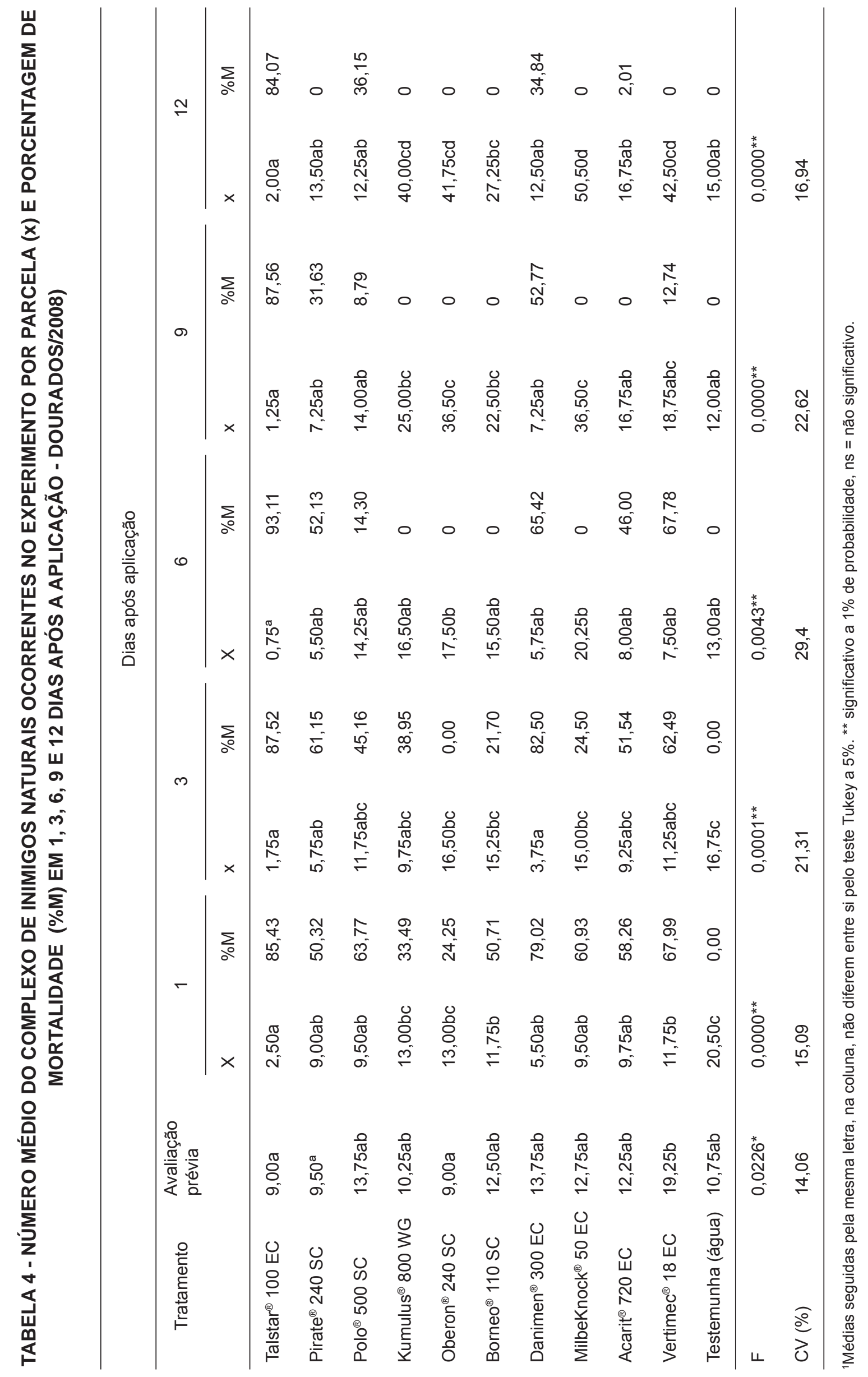


A classificação do IOBCwprs, citada por Boller et al. (2005), aplicada aos tratamentos consta da Tabela 5. A classe foi conferida para o maior grau de toxicidade obtido pelo produto durante o experimento, enquanto a duração da atividade tóxica (em dias) refere-se à mortalidade da população inferior a $50 \%$.

\section{TABELA 5 - CLASSIFICAÇÃO DA SELETIVIDADE A CAMPO DE INSETICIDAS AOS INIMIGOS NATURAIS NO ALGODOEIRO - AVALIAÇÕES DE 1, 3, 6, 9 E 12 DIAS APÓS APLICAÇÃO DE ACORDO COM IOBCWPRS - DOURADOS/2008}

\begin{tabular}{|c|c|c|c|c|c|c|}
\hline \multirow{2}{*}{\multicolumn{2}{|c|}{ Tratamento /Acaricida }} & \multirow{2}{*}{$\begin{array}{c}\text { Dosagem } \\
\left(\mathrm{mL} \text { ou g.ha- }{ }^{-1}\right)\end{array}$} & \multicolumn{2}{|c|}{$\begin{array}{l}\text { Classe da seletividade } \\
\text { (IOBCwprs) }\end{array}$} & \multicolumn{2}{|c|}{$\begin{array}{l}\text { Duração da atividade } \\
\text { tóxica (em dias) }\end{array}$} \\
\hline & & & Aranhas & Scymnus sp. & Aranhas & Scymnus sp. \\
\hline 1 & Talstar 100 EC & 600 & T & $\mathrm{T}$ & $>12$ & $>12$ \\
\hline 2 & Pirate 240 SC & 1000 & M & M & $<6$ & $<9$ \\
\hline 3 & Polo 500 SC & 800 & M & M & $<9$ & $<3$ \\
\hline 4 & Kumulus 800 WG & 600 & M & $\mathrm{N}$ & $<12$ & $<1$ \\
\hline 5 & Oberon 240 SC & 600 & $M$ & $\mathrm{~N}$ & $<3$ & $<1$ \\
\hline 6 & Borneo 110 SC & 230 & M & $\mathrm{N}$ & $<12$ & $<1$ \\
\hline 7 & Danimen 300 EC & 400 & $\mathrm{~T}$ & $\mathrm{~T}$ & $<12$ & $<6$ \\
\hline 8 & $\begin{array}{l}\text { MilbeKnock } 50 \\
\text { EC }\end{array}$ & 240 & M & $\mathrm{N}$ & $<1$ & $<1$ \\
\hline 9 & Acarit 720 EC & 1500 & M & M & $>12$ & $<3$ \\
\hline 10 & Vertimec $18 \mathrm{EC}$ & 600 & M & M & $<9$ & $<9$ \\
\hline 11 & $\begin{array}{l}\text { Testemunha } \\
\text { (água) }\end{array}$ & - & - & - & - & - \\
\hline
\end{tabular}

$\mathrm{N}$ = inócuo, inofensivo ou que não causa danos; $\mathrm{M}$ = moderadamente prejudicial ou moderadamente nocivo; $\mathrm{T}$ = prejudicial, nocivo ou que causa danos.

Com relação à seletividade do acaricida à base de enxofre, Torres et al. (2002) o classificaram como seletivo para o predador Podisus nigrispinus. No presente estudo, esse acaricida foi classificado como seletivo ao Scymnus sp. e ao complexo de inimigos naturais, porém mostrou-se moderadamente prejudicial à ordem Araneae. Tal fato sugere que o produto pode ser também seletivo a outros inimigos naturais, necessitando de estudos específicos. Dentre os acaricidas avaliados, os piretroides Danimen ${ }^{\circledR} 300$ EC e Talstar ${ }^{\circledR} 100$ EC foram classificados como tóxicos (classe T) aos inimigos naturais ocorrentes, concordando com os resultados de Nogueira 
et al. (2007), segundo os quais Danimen ${ }^{\circledR} 300 \mathrm{EC}$ apresentou-se como tóxico aos inimigos naturais ocorrentes na região estudada. A não seletividade observada para os piretróides Danimen ${ }^{\circledR} 300$ EC e Talstar ${ }^{\circledR} 100$ EC pode estar relacionada, segundo Foerster (2002), à similaridade fisiológica do mecanismo de transmissão dos impulsos nervosos do grupo químico, que faz com que os inseticidas neurotóxicos sejam menos seletivos, de modo geral, afetando indistintamente os antrópodes alvo e seus inimigos naturais. Fonseca et al. (2008), avaliando a seletividade de inseticidas aos inimigos naturais ocorrentes sobre o solo na cultura do algodão, citaram Polo ${ }^{\circledR}$ 500 SC como moderadamente tóxico a Araneae, concordando com os resultados obtidos neste estudo. $\mathrm{O}$ inseticida-acaricida Vertimec ${ }^{\circledR} 18 \mathrm{EC}$ foi classificado como moderadamente tóxico aos inimigos naturais observados.

\title{
4 CONCLUSÃO
}

Talstar $^{\circledR} 100$ EC e Danimen ${ }^{\circledR} 300$ EC foram classificados como prejudiciais à Scymnus sp. e Araneae, com duração da atividade tóxica superior a 12 dias. Kumulus ${ }^{\circledR} 800$ WG, Oberon ${ }^{\circledR}$ 240 SC, Borneo ${ }^{\circledast} 110$ SC e MilbeKnock ${ }^{\circledR} 50$ EC mostraram-se inócuos à joaninha Scymnus sp. de acordo com a classificação do IOBCwprs no primeiro dia após a aplicação. Já para Araneae, os produtos desse segundo grupo foram moderadamente prejudiciais. Pirate ${ }^{\circledR} 240$ SC, Polo ${ }^{\circledR} 500$ SC, Acarit $^{\circledR} 720$ EC e Vertimec ${ }^{\circledR} 18$ EC mostraram-se moderadamente prejudiciais para ambos grupos de artrópodes.

\author{
ABSTRACT \\ SELECTIVITY OF ACARICIDES ON Scymnus sp. (Coleoptera: Coccinelidae) AND ARANEAE \\ (Arachnida) IN COTTON (Gossypium hirsutum L.) UNDER FIELD CONDITIONS
}

\begin{abstract}
This study evaluated the selectivity of acaricides used in cotton plantation on predators Scymnus sp. and spiders (Araneae), under field conditions and in accordance with IOBCwprs's classification. Were also studied the percentage of mortality and recovery time of the treatments, through a randomized complete block design, with eleven treatments and four replications. Analysis of the arthropod fauna composition indicated that the most abundant natural enemies occurring in the study were the coccinellid Scymnus $s p$. (58\% of total) and Araneae (24\%). Talstar ${ }^{\circledR} 100 \mathrm{EC}$ and Danimen ${ }^{\circledR} 300 \mathrm{EC}$ were classified as harmful to Scymnus sp. and Araneae, and its toxic activity persisted for more than 12 days. Kumulus ${ }^{\circledast} 800$ WG, Oberon ${ }^{\circledR} 240$ SC, Borneo ${ }^{\circledR} 110$ SC, MilbeKnock ${ }^{\circledR} 50$ CE were harmless to Scymnus sp., and did not show persistence of toxic activity one day after application. Kumulus ${ }^{\circledR} 800$ WG, Oberon ${ }^{\circledR} 40$ SC, Borneo ${ }^{\circledR} 110$ SC, MilbeKnock ${ }^{\circledR} 50$ CE were moderately harmful to Araneae. Pirate ${ }^{\circledR}$ SC 240, Polo $^{\circledast} 500$ SC, Acarit ${ }^{\circledR} 720$ EC and Vertimec ${ }^{\circledast} 18$ EC were moderately harmful to both groups.
\end{abstract}

KEY-WORDS: SIDE-EFFECTS; NATURAL ENEMIES; ACARICIDES; SPIDER; NON-TARGET ARTHROPODS.

\section{REFERÊNCIAS}

1 ANDREI, E. Compêndio de defensivos agrícolas. 7. ed. São Paulo: Organização Andrei Ed. 2005. $1141 \mathrm{p}$.

2 BARROS, R. et al. Avaliação de métodos para quantificar predadores de pragas do algodoeiro. Arquivos do Instituto Biológico, São Paulo, v.70, n.3, p.291-294, 2003.

3 BARROS, R. et al. Flutuação populacional de insetos predadores associados a pragas do algodoeiro. Arquivos do Instituto Biológico, São Paulo, v.73, n.1, p.57-64, 2006.

4 BOLLER, E.F. et al. Working document on selectivity of pesticides. IOBCwprs. Available at: < http:// www.iobc-wprs.org/ip_ipm/03022_IOBC_Pesticide Database_2005.pdf > Accessed on: May 19 2011. 
5 BROWN, K. Non-target arthropod field studies: asking the right questions for their purpose. Pesticides and Beneficial Organisms IOBC/wprs Bulletin, Dijon, v.27, n.6, p.107-108, 2004.

6 CAMPOS, R.A. et al. Artrópodes predadores na cultura algodoeira e comparação de métodos de amostragem. Anais da Sociedade Entomológica do Brasil, Piracicaba, v.15, p.5-20, 1986.

7 CROCOMO, W.B. O que é manejo de pragas. In: CROCOMO, W.B. (Ed.) Manejo de pragas. Botucatu, SP: FEPAF/UNESP, 1984. 240 p. (Curso de Extensão Universitária).

8 FOERSTER, L. A. Seletividade de inseticidas a predadores e parasitóides. In: PARRA, J.R.P.; P.S.M.; BOTELHO, B.S.; CORRÊA-FERREIRA, J.M.S.; BENTO (Eds.) Controle biológico no Brasil: parasitóides e predadores. Barueri, São Paulo: Manole, 2002. p.95-114.

9 FONSECA, P.R.B. et al. Seletividade de inseticidas aos inimigos naturais ocorrentes sobre o solo cultivado com algodoeiro. Pesquisa Agropecuária Tropical, Goiânia, v. 38, n. 4, p. 304-309, 2008.

10 GOMES, F. P. Curso de estatística experimental. São Paulo: Nobel, 1982.

11 GRAVENA, S. Estratégias e táticas de MIP algodoeiro no Brasil. FERNANDES, O.A.; CORREIA, A.C.B.; BORTOLI, S.A. de. (Ed.) Manejo integrado de pragas e nematóides. Jaboticabal: Funep, 1990. p. 1-15.

12 HASSAN, S.A.; HALSALL, N.; GRAY, A.P.; KUEHNER, C.; MOLL, M.; BAKKER, F.M.; ROEMBKE, J.; YOUSEF, A.; NASR, F.; ABDELGADER, H. A laboratory method to evaluate the side effects of plant protection products on Trichogramma cacoeciae Marchal (Hym., Trichogrammatidae). In: CANDOLFI, M.P.; BLÜMEL, S.; FORSTER, R.; BAKKER, F.M.; GRIMM, C.; HASSAN, S.A.; HEIMBACH, U.; MEAD-BRIGGS, M.A.; REBER, B.; SCHMUCK R.; VOGT, H. (Ed.). Guidelines to evaluate sideeffects of plant protection products to non-target arthropods. Montfavet: IOBC/WPRS, 2000. p.107-119.

13 HENDERSON, C.F.; TILTON, E.W. Tests with acaricides against the brown wheat mite. Journal of Economic Entomology, Lanham, v.48, n. 2, p. 157-161, 1955.

14 IRAC. Insecticide resistance Action Committee. Mode of action: classification scheme, v7.0. Available at: < http://www.irac-online.org/wp-content/uploads/2009/09/MoA-classification_v7.0.4-5Oct10.pdf >. Accessed on: May $19^{\text {th }}, 2011$.

15 NOGUEIRA, R. F. et al. Efeito de inseticidas piretróides sobre inimigos naturais das pragas do algodoeiro. In: CONGRESSO BRASILEIRO DE ALGODÃO, 6., 2007, Uberlândia, MG. Resumos... Campina Grande: Embrapa Algodão, 2007. p. 53-55. CD-ROM.

16 NUNES, J. C. S. Ocorrência de inimigos naturais das principais pragas do algodoeiro em Goiás. 1999. 67 p. Dissertação (Mestrado em Agronomia), Universidade Federal de Goiás, Goiânia, 1999.

17 RAMALHO, F.S. et al. Táticas de manejo integrado de pragas em áreas infestadas pelo bicudo-do-algodoeiro. Pesquisa Agropecuária Brasileira, Brasília, v. 25, n. 5, p. 677-690, 1990.

18 RAMIRO, Z.A.; FARIA, A.M. de. Levantamento de insetos predadores nos cultivares de algodão Bollgard DP90 e convencional Deltapine Acala-90. Arquivos do Instituto Biológico, São Paulo, v.73, n.1, p.119$121,2006$.

19 RIBEIRO, J. F. Seletividade de inseticidas aos predadores de pragas do algodoeiro em condições de campo. 2007. 44 p. Dissertação (Mestrado em Produção Vegetal), Universidade Federal da Grande Dourados, Dourados, 2007.

20 SILVIE, P. et al. Manual de identificação dos inimigos naturais no cultivo do algodão. Cascavel: Coodetec/Cirad, 2001, 74 p. (Boletim Técnico, 35).

21 SUJII, E. et al. Comunidade de inimigos naturais e controle biológico natural do pulgão, Aphis gossypii Glover (Hemiptera: Aphididae) e do curuquerê, Alabama argilaceae Hübner (Lepidoptera: Noctuidae) na cultura do algodoeiro no Distrito Federal. Arquivos do Instituto Biológico, São Paulo, v. 74, n. 4, p. 329336, 2007. 
22 TORRES, J. B. et al. Compatibilidade de inseticidas e acaricidas com o percevejo predador Podisus nigrispinus (Dallas) (Heteroptera: Pentatomidae) em algodoeiro. Neotropical Entomology, Londrina, v. 31, n. 2, p. 311-317, 2002. 